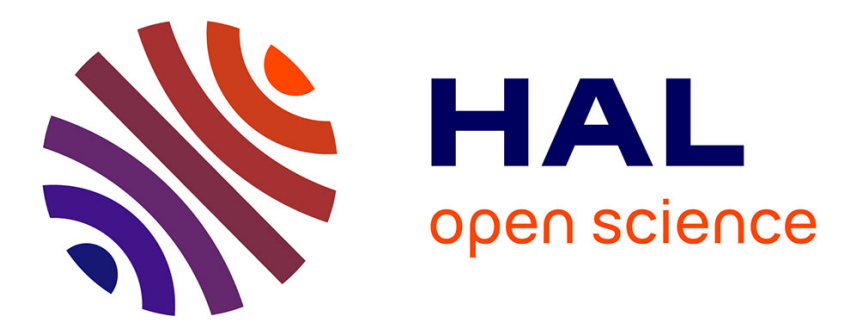

\title{
L'idée d'une science sociale et sa relation à la science politique
}

\author{
Baudouin Dupret, Jean-Noël Ferrié
}

\section{To cite this version:}

Baudouin Dupret, Jean-Noël Ferrié. L'idée d'une science sociale et sa relation à la science politique. Revue Française de Science Politique, 2010, Vol. 60 (6), 1159-1172 [http://www.cairn.info/revuefrancaise-de-science-politique-2010-6-page-1159.htm]. halshs-00548928

\section{HAL Id: halshs-00548928 https://shs.hal.science/halshs-00548928}

Submitted on 20 Dec 2010

HAL is a multi-disciplinary open access archive for the deposit and dissemination of scientific research documents, whether they are published or not. The documents may come from teaching and research institutions in France or abroad, or from public or private research centers.
L'archive ouverte pluridisciplinaire HAL, est destinée au dépôt et à la diffusion de documents scientifiques de niveau recherche, publiés ou non, émanant des établissements d'enseignement et de recherche français ou étrangers, des laboratoires publics ou privés. 


\section{L'IDEE D'UNE SCIENCE SOCIALE ET SA RELATION A LA SCIENCE POLITIQUE}

Peut-on parler de «science sociale »? Partant, peut-on parler de «science politique »? L'étude de phénomènes sociaux et politiques est-elle comptable de méthodologies et de théories comme le seraient, par exemple, les sciences de la nature? S'agit-il, ce faisant, d'expliquer ou de comprendre, de démontrer ou de décrire? En somme, l'épistémologie des sciences sociale et politique vise-t-elle les conditions du discours sur les faits sociaux et politiques ou bien concerne-telle les conditions de production du sens à l'intérieur même de ces phénomènes?

Autant de questions qu'il est possible de tenter d'élucider en s'appuyant sur le livre de Peter Winch, The Idea of a Social Science and its Relation to Philosopby (ISS ci-après), paru en 1958, réédité en 1990 (avec nouvelle introduction) et traduit en français en 2009¹. L'ouvrage, court et lumineux, est dirigé par un impératif de clarification conceptuelle, qui prolonge les travaux de Ludwig Wittgenstein sur les jeux de langage en en montrant les implications sur l'étude des phénomènes sociaux. Souvent mal compris et très généralement sous-estimé2, ISS a été commenté par trois auteurs, liés au mouvement d'ethnométhodologie wittgensteinienne de Manchester ${ }^{3}$, Phil Hutchinson, Rupert Read et Wes Sharrock, qui nous rappellent de façon percutante, dans There is No Such Thing as a Social Science: In Defence of Peter Winch, que les arguments de Winch ne sont pas moins dévastateurs pour la théorie sociale et culturelle d'aujourd'hui qu'ils ne l'étaient pour la sociologie déterministe et ethnocentrique d'il y a cinquante ans.

\footnotetext{
${ }^{1}$ Peter Winch, L'Idée d'une science sociale et sa relation à la philosophie, Paris, Gallimard, 2009 (traduction de The Idea of a Social Science and its Relation to Philosophy, Londres, Routledge, 1990, seconde édition).

${ }^{2}$ Voir. Voir, par exemple, les comptes rendus de H.M. Lubasz dans Ethics, vol. 70, n. 2, 1960, pp. $173-175$ et de P. Alexander dans Philosophy, vol. 34, $\mathrm{n}^{\circ}$ 130, 1959, pp. 278-279 ainsi que le jugement sommaire d'Ernst Gellner dans «Concept and Society » dans B.R. Wilson, dir., Rationality, Oxford, Blackwell, 1970, note 1. La mécompréhension de l'œuvre de Winch est toujours d'actualité : un ouvrage récent revenant sur l'un de ses articles où il critique la façon dont Evans-Pritchard analyse les croyances magiques des Azandé (voir Peter Winch, «Understanding a Primitive Society », dans Peter Winch, Ethics and Action, Londres, Routledge, 1972) le présente comme un «relativiste radical» (voir Pascal Sanchez, La Rationalité des croyances magiques, Paris, Droz, 2007, p. 637-638). On retrouve ici l'erreur rémanente consistant à définir comme « relativiste » le fait de considérer qu'un usage du langage est dépendant d'une forme de vie pour avoir un sens ; pourtant, ce n'est que rappeler une condition pratique pour que celui-ci soit intelligible par des individus forcément situés dans l'espace et dans le temps. Ce que Jean Leca. a pour sa part souligné, précisément à propos de Winch,en notant le refus du naturalisme et l'importance de l'analyse du langage ordinaire lorsqu'il s'agit de décrire les activités humaines (Voir Jean Leca, "Théorie politique», dans Madelaine Grawitz et Jean Leca, dir., Traité de Science politique, Paris, PUF, 1985, p. 121-122).

${ }^{3}$ Phil Hutchinson, Rupert Read et Wes Sharrock, There is No Such Thing as a Social Science: In Defence of Peter Winch, Ashgate, 2008.
} 


\section{La science sociale n'est pas une science}

On ne peut lire Winch sans avoir à l'esprit l'invite sous-jacente de Wittgenstein à grossièrement et simplement regarder et voir, plutôt que toujours intellectualiser et théoriser ${ }^{4}$. Dans ISS, Winch nous invite, s'agissant de l'étude des phénomènes sociaux, à mettre notre façon de penser sur de bons rails et à échapper à ce que Phil Hutchinson et al. nomment, dans leur ouvrage, l' «enchantement des théories ». En ce sens, l'ouvrage ne vise pas à l'élaboration de thèses substantielles, mais à une recherche de clarté, dans une entreprise que l'on pourrait qualifier de déflationniste. A cet égard, on soulignera que la tâche que Winch s'assigne ne tient pas à la découverte d'une information factuelle ou à l'établissement de la véracité d'une proposition empirique, mais au sens (ou au non-sens) de ce que l'on peut dire, dans le monde social et à propos de celui-ci, à l'aide des mots du langage. Il n'est dès lors guère intelligible de se poser la question générale et abstraite du langage (le langage-en-vacance de Wittgenstein) et de sa relation à la réalité, comme si les deux pouvaient être disjoints. Le seul langage auquel on peut s'intéresser, c'est celui qui est en usage. Il en découle la question de l'intelligibilité, non parce que la vérité serait le produit du langage, mais parce que la vérité ne peut procéder que de l'intelligibilité du langagé6.

L'idée d'une science sociale apparait alors comme une simple absurdité, parce que les phénomènes sociaux ne peuvent être traités comme des faits physiques'. L'erreur sous-jacente, ici, tient à ce qu'on suppose que le social puisse être fondé en-dehors de toute intentionnalité, c'est-àdire en-dehors de l'orientation de la pensée humaine vers quelque chose, comme si la réalité sociale pouvait être assimilée à une factualité sans intentionnalité, tant et si bien qu'on pourrait prétendre la décrire indépendamment de son intelligibilité pour les membres de la société. Il n'est pourtant pas possible de faire des recherches sur des «objets» tels que le «comportement social» comme s'il s'agissait d'un « fait brut $»^{8}$ (apportant une preuve empirique) et non d'un concept (dont l'usage doit être élucidé) $)^{9}$.

Plutôt que de "science sociale», il conviendrait donc, sans doute, de parler d'étude des phénomènes sociaux, étude s'assignant la tâche de clarifier les phénomènes d'intelligibilité. En ce sens, il s'agit d'entreprendre des investigations conceptuelles, où les « concepts » étudiés sont ceux des gens dont on analyse l'activité (en lieu et place d'une élaboration conceptuelle professionnelle). Dans l'ordre des actions humaines, en effet, la factualité est inséparable de la commune intelligibilité, ce qui implique que l'idée d'une science sociale parente des sciences de la nature (et, partant, d'une épistémologie parente de l'épistémologie de ces sciences) est un dévoiement par rapport à ce dont est faite la vie sociale. De ce point de vue, les sciences sociales apparaissent quoiqu'elles prétendent - comme une philosophie, cette sorte de philosophie opaque dénoncée par Wittgenstein, une philosophie empêtrée dans ses « grandes questions ».

L'erreur vient sans doute de ce que l'on imagine que notre participation en tant qu'individus dans la société doit être pensée comme une relation de détermination causale, la causalité étant ici entendue dans l'acception que lui ont donnée Hume et Mill, c'est-à-dire dans son sens scientifique, celui d'un rapport entre des «objets» et non entre des intentions ${ }^{10}$. Deux choses doivent donc être soulignées : d'une part, ce type de causalité n'est pas adéquat au monde social ; de l'autre, comme le souligne Winch dans sa préface à la réédition d'ISS, le monde social développe ses

\footnotetext{
4 Voir L. Wittgenstein, Investigations philosophiques, \126 (mais aussi \38), Paris, Gallimard, 1961 : «La philosophie place seulement toute chose devant nous, et n'explique ni ne déduit rien. - Puisque tout est étalé sous nos yeux, il n'y a rien à expliquer »; voir aussi, Phil Hutchinson et al., op. cit., p.91 et suivantes.

${ }^{5}$ Phil Hutchinson et al., op. cit, p.91

${ }^{6}$ Voir L'idée d'une science sociale... op. cit., p. 64 et suivantes, notamment p. 67 : «Notre idée de ce qui appartient au royaume de la réalité nous est donnée dans le langage que nous utilisons »

${ }^{7}$ Ibid. p. 50 : «Je me propose dans cette monographie d'attaquer cette conception de la relation entre les sciences sociales, la philosophie et les sciences de la nature »

8 Pour reprendre la distinction formulée par John Searle entre «faits bruts » et « faits institutionnels » (voir John Searle, La Construction de la réalité sociale, Paris, Gallimard, 1995, p. 45 et suivantes.

${ }_{9}$ Voir notamment L'idée d'une science sociale... op. cit., p. 60, p. 70 ; voir aussi, p. $77:$ «... les relations sociales sont des expressions des idées touchant la réalité ».

${ }^{10}$ Ibid., p. 68 et suivantes ; p. 145 et suivantes.
} 
propres jeux de langage causaux ${ }^{11}$. Si la sociologie s'impose, par conséquent, ce n'est pas en tant que science de la causalité sociale, mais comme activité ordinaire de tout un chacun, dans les formes de vie qui lui sont propres, avec les jeux de langage (y compris causaux) qui lui conviennent. Il ne peut donc être question d'explication causale des phénomènes sociaux, mais uniquement de compréhension et de description. Comprendre, c'est saisir le sens, ce qui est une réalisation, un aboutissement, comme l'a montré Wittgenstein ${ }^{12}$. Comprendre un phénomène social, c'est saisir des régularités dans les façons de comprendre des êtres humains, ce n'est pas saisir ce qui se passe indépendamment de la manière dont ils le comprennent et dont, le comprenant, ils le produisent. A cet égard, il faut insister sur le fait que les problèmes de compréhension académiques et ordinaires sont du même ordre ; à vrai dire, la compréhension académique présuppose nécessairement la compréhension ordinaire. Le problème vient de notre tradition intellectuelle pour laquelle comprendre serait le produit d'une méthode universelle, alors même qu'il n'existe pas de formule pour comprendre : comme le dirait Wittgenstein, l'aube se lève sur un objet, celui-ci prend forme, il « naît $"\left(\right.$ comme le jour peut « naitre ») ${ }^{13}$.

\section{Etudier les phénomènes sociaux est une réflexion conceptuelle}

Parce que c'est au principe même de la différence entre sciences physiques et études des phénomènes sociaux, nous devons distinguer les questions empiriques des questions conceptuelles ${ }^{14}$. Ainsi, la question de savoir si les dodos de l'île Maurice «existent» est-elle une question empirique, dont la réponse est dotée de critères permettant de dire «ce qui compte comme » un dodo, l'île Maurice et l'existence. En revanche, la question de savoir si le monde existe au-delà de mes pensées ne peut être résolue par la méthode expérimentale; c'est une question conceptuelle sur la place du concept de réalité dans la vie de ceux qui vivent avec ce concept, une question relative à des finalités propres à ces gens et sensible à des circonstances dont on ne peut les abstraire (sous peine de la détacher de ce qui en fait l'identité). De même, la question de savoir s'il existe des actes vraiment altruistes est-elle conceptuelle, en ce sens qu'on ne peut y répondre en se fondant sur des critères susceptibles d'un accord in abstracto, mais seulement en la rattachant aux circonstances et finalités spécifiques au contexte auquel elle se rattache.

Décrire, quand on s'attache à étudier les phénomènes sociaux, c'est décrire des actions, non des mouvements. De plus, comme le rappellent opportunément Phil Hutchinson $e t$ al., « action » ne doit pas être entendu au sens consacré par la sociologie, celui d' "action sociale », qui sous entend que l'action se comprend par rapport à une structure sociale et à une théorie de la structuration du social ${ }^{15}$. Action doit s'entendre de manière également déflationniste comme un " agir sensé », ce qui implique de concevoir l'action en relation avec la structure d'intelligibilité spontanément mise en œuvre par les membres et non avec la structure sociale conceptualisée par les sociologues. C'est ainsi que, si nous décrivons quelque chose comme l'action de donner un coup de poing, nous avons nécessairement inclus les intentions de la personne qui a frappé, faute de quoi nous n'aurions décrit qu'un geste. Autrement dit, la description d'une action est nécessairement intensionnelle/intentionnelle. Ou encore, si nous disons de quelqu'un qui se lave les mains qu'il fait ses ablutions rituelles, nous décrivons, en incluant l'intention qui accompagne son geste, son action. La question de l'identité d'une action, c'est-à-dire ce qui fait que ce geste rentre sous une appellation (ce geste est-il un coup de poing ou un rite d'ablution), n'est pas une question empirique, mais bien conceptuelle. La réponse qui sera apportée à cette question ne pourra pas non plus être falsifiée empiriquement (le geste décrit restera toujours le même). Et si la signification de ce geste nous semble incertaine, nous porterons un jugement sur les relations, internes, qui l'unissent aux

11 Voir. L'idée d'une science sociale... op. cit., p. 38 et suivantes.

12 L. Wittgenstein, Investigations..., op.cit., par exemple. \ 154 : «Essayez de ne pas du tout penser à la compréhension en tant qu'à un 'processus psychique'»; et $\int 153$ : «Et comment le processus de la compréhension pouvait-il rester caché, alors que je disais 'Maintenant je comprends' parce que j'avais compris ».

${ }^{13}$ L. Wittgenstein, Investigations..., op.cit., p. 326 : «Et je dois faire la distinction entre la 'vue constante' d'un aspect et sa 'naissance'».

${ }_{14} \mathrm{Ph}$. Hutchinson et al., There is No Such Thing..., op.cit., pp. 75-79.

${ }^{15}$ Ibid., p. 95 et suivantes 
intentions de son auteur. Par internes, il faut entendre que ces relations ne concernent pas deux entités distinctes, mais plutôt des choses qui vont de pair l'une avec l'autre, qui "vont ensemble » (p.ex. «brûler », qui va de pair avec « feu »).

Dans cette démarche, il ne s'agit pas d'atteindre ce qu'il y a au-delà du langage, mais bien ce qu'il y a dans le langage. Le mot "vérité » n'est donc, ici, qu'un mot comme les autres, dont les critères d'évaluation ou de vérification varient avec les usages. Autrement dit, il convient d'identifier le jeu de langage dans lequel l'énoncé de vérité est formulé pour savoir à quoi cet énoncé répond (ses critères d'intelligibilité). Notons qu'il ne faut pas comprendre les jeux de langages comme les pièces d'une théorie, encore moins comme des équivalents de concepts holistiques tels que des cultures ou des institutions, de sorte que s'y référer n'implique nullement l'adoption d'une posture relativiste ${ }^{16}$. Notons aussi que cela ne revient pas à soutenir que la réalité serait une construction sociale: les gens ne créent pas la réalité par le langage; ils s'orientent vers la réalité à travers les descriptions de celui-ci. On peut donc dire que le langage et la réalité sont reliés de façon interne. Pour Winch comme pour les wittgensteiniens, la réalité n'est donc tout simplement pas un problème d'interprétation, c'est-à-dire qu'elle ne réside pas dans les conceptualisations ${ }^{17}$

\section{Décrire plutôt qu'interpréter}

Mener une élucidation conceptuelle, c'est tout l'inverse d'une montée en généralité réductionniste. L'idée de «science» sociale est, pour sa part, toute entière construite sur un réductionnisme, qu'il soit méthodologique ou substantiel ${ }^{18}$. Et ce réductionnisme suppose l'apriorisme, c'est-à-dire l'adhésion à une méthode ou à une explication avant examen. C'est vrai du scientisme et de l'attitude théorisante, mais aussi du postmodernisme qui, en dépit de son refus d'une théorie générale, n'en est pas moins le produit d'une déduction théorique basée sur l'idée que le langage est un système auto-contenu. Tous ces courants des sciences sociales ont en commun de prétendre fournir une lentille méthodologique. Autrement dit, ils sont massivement préoccupés par les formes de l'explication ${ }^{19}$. Mais là où les grandes théories sociales se disputent pour savoir quel est le meilleur schéma d'explication du social, Wittgenstein et Winch entendent dissiper le sortilège intellectualiste en vertu duquel le tandem théorie-et-méthode serait un précurseur essentiel à la compréhension. A vrai dire, c'est précisément de l'adoption de pareilles conceptions a priori de ce que comprendre doit être que surgissent les obstacles à la compréhension. Il convient donc de se déprendre de l'idéal des sciences sociales, celui d'une compréhension extérieure, unique, réflexive et englobante. A tout le moins, il faut être prudent quant au besoin de théorie. Il existe, en effet, d'autres formes de compréhension que théoriques (sauf à entendre théorie d'une manière tellement extensive que cela ne désigne plus rien de spécifique, voire plus rien du tout, comme c'est le cas de cette tendance à crier à l'automystification de celui qui nie avoir une théorie).

En lieu et place d'une science sociale dont l'aspiration à la généralité absorbe les cas empiriques, l'étude des phénomènes sociaux que suggèrent Wittgenstein et Winch - et que pratique l'ethnométhodologie - considère le cas empirique comme une fin en soi, cherchant à décrire comment des activités spécifiques sont enchâssées dans les environnements sociaux auxquels elles appartiennent et dans les pratiques qu'elles incarnent. Dans cette démarche, il n'existe plus de raison d'accepter que le sociologue soit qualifié pour écarter les critères indigènes de détermination de l'identité des actions. Bien au contraire, ces critères sont établis dans le contexte dans lequel ces actions sont menées.

\footnotetext{
${ }^{16}$ Le relativisme est la position normative selon laquelle, d'une part, les cultures et les institutions se valent et, d'autre part, la réalité se mesure à l'aune des valeurs du groupe ou de la société considérée. Cette affirmation est elle-même normative. Le relativisme est donc contradictoire de ce point de vue, puisqu'il refuse la domination d'un ordre normatif mais que ce refus est lui-même normatif. Les positions de Wittgenstein et de Winch ne sont pas normatives (en ce sens), dans la mesure où elle ne portent pas sur les valeurs mais sur le fait que les choses ne peuvent se comprendre en dehors de leur contexte d'énonciation.

${ }^{17}$ Ibid., p. 69.

${ }^{18}$ Ibid., p. 2-5.

${ }^{19}$ Ibid., p. 93.
} 
En dégageant l'étude des phénomènes sociaux de l'obsession de la méthodologie et en les recentrant sur l'identification et la compréhension de l'action, l'on peut retrouver une vue claire de ce qui est ordinairement compris par tout un chacun, dans le cours ordinaire de la vie, du fait de la familiarité qui est entretenue, par la personne observée comme par l'observateur, avec les modes pratiques de conduite des choses. En ce sens, celui qui étudie les phénomènes sociaux n'est pas tant un observateur scientifique extérieur à ce qu'il observe qu'un membre de ce monde social s'appropriant les façons de comprendre qui sont propres à ses habitants. S'approprier ces modes de compréhension, ce n'est pas les interpréter, dans la mesure où on n'interprète que quand on n'a pas saisi le sens. On est loin de toute idée de découverte d'une chose ignorée, de choix entre des hypothèses alternatives, d'interprétation d'un phénomène qui nous reste obscur; il s'agit bien davantage d'une description, c'est-à-dire d'une présentation perspicace. Il existe une distinction ordinaire entre saisir et interpréter. Nous n'appelons pas toutes nos saisies du monde des interprétations. Quand la science sociale s'engage dans cette direction, elle ne contribue pas à la compréhension de notre monde, mais, tout au contraire, elle obscurcit notre façon de le rencontrer.

Décrire, ce n'est ni interpréter, ni expliquer, ni comprendre au sens wébérien (Verstehen). Cela n'empêche pas qu'en décrivant ce que les gens font, on puisse fournir une explication de ce qu'ils font, pourvu que ce soit précisément fait en connexion avec ce qu'ils font. Mais, au principal, il ne s'agit pas tant de créer l'impression qu'il y a quelque chose qui doit être expliqué que de procéder à la description perspicace de phénomènes qui ne sont pas dans l'attente de leur explication. Cette description ne peut faire l'économie de la contextualisation, c'est-à-dire du rappel des critères locaux d'identification des actions qui sont menées. Pour dire les choses autrement, les critères d'identité n'appartiennent pas au sociologue, mais bien aux contextes sociaux dans lesquels ces actions prennent place. Par conséquent, les modes corrects de description d'une action dérivent des pratiques dans lesquelles les gens sont engagés, étant entendu que les critères d'une description correcte sont ceux qui sont appliqués par les participants compétents à ces pratiques. Ainsi, identifier correctement la mort de quelqu'un en tant que suicide ne peut procéder d'une comptabilité sociologique indépendante des déterminations propres au médecin légiste, entre autre. Ce qui revient à dire que les identifications correctes sont celles qui sont appropriées aux pratiques propres à un environnement.

\section{L'idée de science politique : tout est-il ou n'est-il pas politique?}

L'approche de Winch concerne aussi la science politique, en tant qu'elle entend à la fois être une «science » et, plus largement, s'inscrire dans le domaine des «sciences sociales». Revenir sur une récente controverse permettra certainement de comprendre tout le parti qu'il est possible de tirer du refus de considérer l'étude du social comme une « science » et, inversement, dans quelles erreurs conceptuelles nous entraîne la volonté de le faire. Nous faisons, bien sûr, référence à la controverse qui a opposé Bruno Latour et Pierre Favre à propos du domaine de la science politique et des science studies ${ }^{20}$. Pour le dire rapidement - tout au moins sans revenir en détail sur les positions de chacun - Bruno Latour soutient que «la politique est partout» et, notamment, dans l'activité proprement technique de la science ${ }^{21}$. Preuve en serait qu'en partant de la physique nucléaire, on peut en arriver aux dangers que la détention des bombes atomiques font peser sur l'humanité ou qu'en transformant les cadres de perception de l'espace et du temps, la relativité modifierait «les fondements mêmes de l'existence commune $»^{22}$. Pierre Favre objecte que "tout n'est pas politique ", au sens où tout n'est pas dans tout ${ }^{23}$. Nous avons donc, d'un côté, un protagoniste affirmant que le politique est partout et, de l'autre, son contradicteur affirmant que l'extension du politique n'est pas infinie. On ne saurait imaginer plus fort contraste. Pourtant, d'un point de vue winchien, ces deux positions sont assez similaires. Affirmer que le politique est ou n'est pas partout,

20 Voir Bruno Latour, "Pour un dialogue entre science politique et science studies », Revue française de science politique vol. 58, n4, 2008 et Pierre Favre, «Ce que les sciences font à la science politique. Réponse à Bruno Latour », Revue française de science politique, vol. $58, \mathrm{n}^{\circ} 5$.

${ }^{21}$ Bruno Latour, art. cité, p. 661

${ }^{22}$ Ibid., p. 661-662.

23 Pierre Favre, art. cité, p. 821-822. 
c'est, en effet, considérer qu'il existe a priori un domaine d'extension du politique et que cette extension peut-être discutée d'un point de vue disciplinaire. En d'autres termes, il est possible, pour Bruno Latour comme pour Pierre Favre, de qualifier des phénomènes de politiques d'un point de vue disciplinaire, qu'il s'agisse des science studies ou de la science politique. D'un point de vue winchien, cette qualification n'est possible que dans la perspective des critères d'identification locaux, ceux des personnes impliquées dans un cours d'action, et non du point de vue des professionnels, qui auront tendance - comme le montre, du reste, la controverse Latour/Favre - à user des critères tirés de leurs propres activités.

Prenons un exemple tiré de Winch lui-même : si $\mathrm{N}$ vote pour un gouvernement soutenant des travaillistes afin de préserver la paix industrielle, l'explication de son vote par le but poursuivi n'a de sens que si $\mathrm{N}$ a une idée même vague de ce que signifie « préserver la paix industrielle » et de la connexion entre celle-ci et la politique conduite par le gouvernement si les travaillistes sont élus. $\mathrm{Si} \mathrm{N}$ ne sait pas ce que signifie «préserver la paix industrielle » et s'il vote tout de même travailliste et que ceux-ci sont élus, la mise en relation du résultat et de l'impératif de préserver la paix industrielle n'a aucun sens ${ }^{24}$. L'action de $\mathrm{N}$ ne peut être expliquée indépendamment de la compréhension de ce qu'il fait. S'il ne sait pas qu'il vote, il n'y a pas de sens à dire qu'il vote et s'il ne sait pas ce qu'est la "paix industrielle ", il n'y a pas de sens non plus à attribuer un éventuel succès des travaillistes à un choix en faveur de la "paix industrielle». Un exemple semblable figure chez Hilary Putnam. Il remarque qu'une fourmi qui dessinerait, dans son parcours sur le sable, une forme semblable au profil de Winston Churchill ne pourrait pour autant être décrite comme ayant dessiné le portrait de Winston Churchill25. En d'autres termes, ce qui permet de qualifier l'action, c'est l'intention des membres.

La "politisation", sur laquelle on a beaucoup écrit et qu'évoque, de fait, Bruno Latour à propos de la lettre d'Einstein, n'a de sens que si ce que les gens sont en train de faire leur apparait comme politique ou que ce qu'ils font est perçu par d'autres comme tel (et encore n'est-ce politique que depuis leur perspective). Leur qualification est souveraine. Il en est de la politique comme de la religion chez Nadel, qui considère comme « religieux » ce que les membres de la société considèrent comme tels ${ }^{26}$. Pour les sciences naturelles, il pourrait exister du «religieux» sans intentionnalité, comme il existe du calcaire, des protons ou du bois. Mais la politique est une activité conceptuelle, au sens de Winch ; elle n'existe que si l'on pense que l'on en fait. La question se pose, bien sûr, de la politique à huis clos. Si je parle politique dans ma cuisine et que je suis conscient de parler politique, peut-il s'agir de politisation ? Ici aussi, le langage de «l'homme de la rue » ${ }^{27}$ est un guide sûr : dans ma cuisine, « je parle politique » comme je pourrais en parler accoudé au comptoir d'un café. Parler politique, ce n'est pas en faire. Si la conséquence de ces propos est que je décide de participer à une manifestation contre le gouvernement, j'ai le sentiment de faire de la politique et d'en faire collectivement; c'est d'ailleurs, l'idée et le fait d'entrer dans un collectif d'un certain type qui me permettra de me dire que je fais une action politique. Le collectif ou le «commun » ne sont pas des objets philosophiques, mais des conditions pratiques pour faire de la politique comme au moins un ballon, un but et un gardien sont des conditions nécessaires pour que des enfants puissent dire qu'ils jouent au football. Ce ne sont pas pour autant (nous y reviendrons rapidement) des acteurs non humains, au sens de Latour. Concluons d'abord sur la politisation. Le terme introduit un faux problème : savoir si oui ou non ce que l'on fait est " politisé ». C'est un faux problème, parce que les gens ont déjà qualifié, au moins pour eux-mêmes, ce qu'ils font et qu'ils savent bien que, quand ils parlent dans leur cuisine, ils ne font pas de la politique, mais en parlent - et que, quand ils se joignent à une manifestation contre le gouvernement, ils en font. De ce point de vue, théoriser la politisation,

\footnotetext{
${ }^{24}$ Voir , L'idée d'une science sociale... op. cit., p. 107 et suivantes.

25 Voir Hilary Putnam, Raison, vérité et histoire, Paris, Minuit, 1984, p. 11 et suivantes.

26 Voir Siegmund Frederick Nadel, Nupe Religion. Traditional Beliefs and the Influence of Islam in a West African Culture, Londres, Routledge et Kegan Paul, 1970 [1954]. C'est le même argument que nous tenons, à propos du droit islamique, dans «L'Autorité de la référence. Usages de la sharîa islamique dans le contexte judiciaire égyptien », Archives de sciences sociales des religions, 49e volume, n 125, pp. 189-209.

27 Il s'agit d'une expression de John Austin dans un texte où il souligne la richesse dénotative du langage ordinaire (voir John Austin, Le Langage de la perception, Paris, Vrin, 2007, p. 79, p. 83 et suivantes).
} 
c'est rentrer dans cet «enchantement de la théorie » pointé par Phil Hutchinson et al. qui nous empêche de voir les choses pour elles mêmes ${ }^{28}$.

La question peut se poser, bien sûr, de manifestants qui demandent, par exemple, que le repos dominical soit respecté afin que l'on puisse consacrer ce jour à Dieu. Ces manifestants soutiennent, en même temps qu'ils formulent leur revendication, que leur cause n'est pas politique. De quel point de vue peut-on prétendre qu'elle l'est? Si, par exemple, la manifestation n'a aucune conséquence et passe aussi inaperçue qu'une conversation politique à la cuisine, qu'est-ce qui pourra permettre de dire qu'elle est politique plutôt que religieuse? Le fait qu'elle s'adresse aux gouvernants ? Il faudrait introduire le critère : «s'adresser aux gouvernants » comme marqueur systématique de politisation, mais pour qui s'adresser aux gouvernants est-il politique, si ça ne l'est pas du point de vue de ceux qui le font? Dans le cas qui nous occupe, ça ne peut être que du point de vue de l'observateur professionnel, disons le politiste, décidant que c'est politique. A son tour, il accède à la souveraineté de la qualification, mais cette souveraineté ne contribue pas à enrichir notre compréhension des phénomènes; elle se borne à délimiter deux «mondes» incommensurables : celui des manifestants pour qui l'action n'est pas politique et celui du politiste pour qui l'action est politique. La qualification du politiste est-elle fondée empiriquement? Non, puisque l'attribution d'un caractère politique à la manifestation découle du critère qu'il a introduit et non de la matérialité du phénomène lui-même. Au contraire, les êtres placés au cœur du phénomène disent que ce n'est pas politique. «Politique » n'est donc pas un fait brut ; c'est tout simplement un concept, qui plus est détaché de l'expérience, contrairement à la conceptualisation des manifestants pour qui ce n'est pas politique, et qui est, elle, intrinsèquement liée à l'expérience.

L'un des problèmes de la «méthode scientifique » est donc, comme nous le voyons, qu'elle conduit à confondre les concepts des observateurs avec des faits bruts. Admettons, maintenant, que les gouvernants dénoncent la manifestation comme politique, qu'il s'en ensuive une polémique avec ses organisateurs et que les partis d'opposition (tout au moins certains) s'en saisissent. Il y aura «politisation » du fait des gouvernants. La politisation découlera, non de l'action des manifestants (et encore moins de leurs intentions), mais de la qualification que lui font subir les gouvernants. Cette qualification ne transformera pas magiquement la manifestation en fait politique, sauf si les organisateurs décident de la faire leur, de même que la controverse ne sera pas, par elle-même politique. Là où il existe une tendance marquée à confondre le collectif, le public et le politique, on se contentera de remarquer que, d'un point de vue winchien, définir extérieurement la controverse comme politique, c'est, une fois de plus, conceptualiser ce qui se passe à la place des gens concernés. Ceci apparait d'autant plus frappant que la controverse porte précisément sur le fait de savoir si, oui ou non, une chose est politique, de sorte que, tenir pour acquis que cette discussion est déjà politique revient à considérer la discussion comme inutile, alors même qu'elle a lieu et qu'elle paraît nécessaire aux différentes personnes impliquées. Une controverse publique est d'abord une controverse, avant d'être éventuellement politique (ou littéraire, ou scientifique, ou n'importe quoi d'autre), comme une dague est l'arme du crime ou un simple presse-papier en fonction de l'usage qu'on en fait, c'est-à-dire de l'intention avec laquelle on en use. Considérer qu'une controverse est forcément politique, c'est partir d'une définition de la politique comme controverse pour dire ce qu'est une controverse. Mais c'est alors la définition de la politique que l'on donne et non celle de la controverse.

\section{Le vertige du point de vue surplombant}

Dans le même ordre d'idée, on remarquera que l'extension maximale du politique - «le politique est partout» - que propose Bruno Latour suppose une définition préalable, en quelque sorte surplombante, en vertu de laquelle serait politique tout ce qui implique l'action en commun. Or le ciment collectif, ce qui fait que nous partageons indéniablement le même monde, n'est en rien politique (pas plus que ne l'est l'air que nous respirons tous). Nous vivons dans un monde commun parce que nous en partageons l'intelligibilité, c'est-à-dire les mécanismes pour lui donner un sens, et

${ }^{28}$ Voir Ph. Hutchinson et al., There is No Such Thing..., op.cit., chapitre 3 : «Seeing Things for Themselves : Winch, Ethnography, Ethnomethodology and Social Studies ». 
qu'il en résulte ce que Schütz nomme une perspective réciproque ${ }^{29}$. Confondre nos dispositifs d'intellection avec des dispositifs politiques, c'est nommer une réalité par le nom d'une autre. Bien sûr, on pourra dire, en se référant aux Grecs, que le logos est politique, mais c'est là aussi une question conceptuelle et non une question empirique. C'est l'opinion que nous prêtons aux Grecs en la tirant de l'un ou l'autre de leurs philosophes. Nous ne savons même pas si c'est ce que n'importe quel Grec croyait ${ }^{30}$. Nous nous servons routinièrement de méthodes communes pour rendre le monde intelligible sans que cela ne requière la moindre once de politique. Assister à la projection d'un film implique ainsi la reconnaissance d'un cadre commun, la redéfinition momentanée de la conception de l'espace et du temps, un arrière-plan de compréhension permettant d'accepter des raccourcis narratifs, comme le fait que la continuité d'une scène à l'autre est admise encore que jamais montrée ${ }^{31}$. C'est ainsi qu'un personnage ouvre la porte et marche dans la rue, sans qu'on l'ait vu quitter l'appartement ni descendre l'escalier, ou qu'un autre commence à relater l'histoire de sa vie dans une pièce et que, l'instant d'après, on voit celle-ci se dérouler sous nos yeux avec le même personnage instantanément rajeuni. Dix ans d'une vie peuvent alors se passer en un seul quart d'heure et l'on admet qu'il est possible de visionner le passé.

Ceci n'a rien de politique. C'est le travail de notre esprit, lequel travaille en s'appuyant sur des dispositifs extérieurs. Personne ne dira qu'il fait de la politique quand il se repère par rapport au passé ou se projette dans le futur, quand il se situe sur une carte comme quand il regarde les étoiles avec un ami. Certes, ces actions (et d'autres) peuvent devenir politiques mais, en le devenant, elles ne révèlent pas qu'elles l'étaient préalablement; elles le deviennent parce que les membres les accomplissent intentionnellement et explicitement comme politiques par référence à un contexte qui soutient cette qualification. On peut très bien imaginer le président Kennedy regardant la lune comme un enjeu de compétition entre les Etats-Unis et l'Union soviétique et le même président la contempler, le lendemain, en chantonnant «Fly me to the Moon ». La lune, en tant que fait brut, n'a pas cessée d'être la lune, une étoile morte tournant autour de la terre; ce qui s'est modifié, c'est le contenu sémantique de la référence, en d'autres termes l'intentionnalité. De ce point de vue, la lune, les fusées et les ordinateurs qui permettent de contrôler les fusées ne sont en rien des acteurs non humains ; ils sont, certes, non humains, mais ce ne sont pas des acteurs, tout simplement parce qu'ils sont entièrement dénués de la possibilité de faire référence à quoi que ce soit. Ils existent, bien sûr, mais en tant que contrainte contextuelle collectivement mise en œuvre. On ne peut pas faire référence à une fusée en tant que candélabre, non pas parce que la fusée s'y opposerait, mais parce que des êtres humains le feraient. Notre correction par rapport au langage (comme par rapport aux règles) provient, en effet, ainsi que le montre Winch à la suite de Wittgenstein, de ce que nous sommes soumis à l'obligation d'être intelligibles pour autrui ${ }^{32}$. C'est la réaction d'autrui qui nous dit si notre langage est correct, ce ne sont pas les objets. Ce qui vaut pour les fusées vaut pour les pompes à air, auxquelles se réfère Bruno Latour. Ni les unes ni les autres ne sont donc intrinsèquement politiques; elles sont ce que les formes de vie et les jeux de langages qui en relèvent permettent d'en faire. Et, contrairement à ce que suggère Pierre Favre, ces formes de vie et ces jeux de langage n'impliquent nullement une posture relativiste. Ils constituent, au contraire, une contrainte collectivement mise en œuvre à partir de l'évidence commune des faits bruts (la lune, la fusée, etc.).

Comme on le voit, suivre la qualification des membres plutôt que la considérer préalablement déterminée ne nous fait rien perdre des phénomènes; bien au contraire, suivre la qualification fait apparaitre la complexité des phénomènes, complexité qui n'est pas d'un seul tenant, mais qui vient plutôt de ce que les mêmes objets du réel peuvent acquérir des statuts différents en fonction de ce que nous faisons. Ils ne sont donc pas des acteurs potentiellement politiques, des non-humains appartenant à un monde commun, mais des êtres que l'on se représente différemment en fonction des contextes. Se disputer pour définir a priori le domaine du

${ }^{29}$ Voir Alfred Schütz, Le Chercheur et le quotidien. Phénoménologie et sciences sociales, Paris ,Klincksieck, 1992.

$30 \mathrm{Ou}$ à quoi croyaient exactement les Grecs. Voir Paul Veyne, Les Grecs ont-ils cru à leurs mythes? Paris, Seuil, 1983.

31 Voir Lena Jayyusi, «Toward a socio-logic of the film text », Semiotica, vol. 68, n³-4, 1988

32 Voir L'idée d'une science sociale... op. cit., p. 78 et suivantes, notamment, p. 86 : «...je ne puis, de façon intelligible, être dit suivre une règle que dans une situation au sein de laquelle quelqu'un d'autre pourrait en principe découvrir la règle que je suis ». 
politique est une question que Wittgenstein dirait dénuée de sens ; le qualificatif "politique» émerge, tout simplement, des actions qui s'orientent vers ce domaine en tant que tel, et ce de manière non problématique. En un mot, le politique n'est pas effectivement partout; il peut seulement être n'importe où, dès lors que l'on s'entend pour l'y trouver et dès lors que c'est contextuellement sensé. Mais ce mécanisme là n'est pas politique ; ce mécanisme là, c'est le travail social de l'esprit.

Il est clair - quand bien même ne le partagerait-on pas - que le point de vue winchien contient ainsi une solution élégante et robuste au débat rémanent sur ce qui est ou n'est pas politique, en même temps qu'il permet de sortir des faux problèmes introduits par la notion théorisante de "politisation » : celle-ci, considérée en tant que phénomène, émerge tout bonnement, de manière non interprétative, des qualifications et orientations accompagnant les cours d'action. Le fond de la question est de ne pas oublier que les gens observés par le politiste ne sont pas des électrons, des pierres ou des robots, mais des individus dont l'identité est produite de manière endogène au fil de l'interaction, et que le politiste flotte dans la même eau qu'eux.

\section{L'erreur scientiste : partage disciplinaire et réel partagé}

L'erreur scientiste que dénonce Winch n'est donc pas une erreur liée à un certain type d'épistémologie, puisque des épistémologies aussi dissemblables que celles de Bruno Latour et de Pierre Favre conduisent finalement au même résultat, qui est de considérer le domaine du politique comme antéprédicatif, c'est-à-dire comme pouvant être déterminé indépendamment des actions spécifiques qui s'y réfèrent. Cette erreur nous apparait donc liée à une conception bien plus large, résidant dans l'idée, inspirée des sciences de la nature, que ce qui est social doit, pour exister, exister indépendamment de l'esprit des gens. Il en découle la mise en place d'une ontologie tout à la fois restrictive, réductionniste et erronée, ne tenant pas compte des "objets fictifs », quoique traitant certaines entités conceptuelles comme empiriques, à l'instar de ce que Durkheim fait à propos de la société. La règle s'appliquant à ces ontologies semble être que l'existence implique nécessairement un même mode d'être. Il en découle que l'Etat apparait difficile à penser sociologiquement, puisque, pour le penser, il faut alors le considérer comme matériel et que ne pas le penser, au motif qu'il n'est pas matériel, reviendrait à faire l'impasse sur un fait majeur de la vie politique. C'est ce que Dominique Linhardt nomme «l'embarras de la sociologie de l'Etat $»^{33}$.

Son texte montre, de manière « documentée », que l'Etat ne peut être considéré ni comme un « être à existence erronée » ni comme un être empirique. Toutefois, cette monstration, à l'instar de celles conduites par Bruno Latour et Pierre Favre, est menée depuis une posture épistémologique, l'élucidation de la structure de la réalité, se fondant, de nouveau, sur une discussion interne à un domaine disciplinaire - ici, la sociologie. C'est à partir de l'examen critique d'anciennes conceptualisations d'observateurs que s'opère la reconception de la question ontologique de l'Etat. Le problème intrinsèque de cette approche est qu'elle ne traite pas réellement de ce dont elle prétend traiter, puisqu'elle parle de la « littérature-sur » plutôt que du phénomène luimême. Elle relève de ce que Garfinkel nomme le «quelque-chose qui manque » (missing-what) ${ }^{34}$, par quoi il vise l'oubli de ce qui est exactement en question et que l'on pourrait désigner, à la suite de l'injonction que nous adresse Louis Quéré de "rendre justice aux phénomènes", comme le "phénomène » dont il s'agit de rendre compte. Or, le phénomène dont rend compte ${ }^{35}$ l'étude des « embarras de la sociologie de l'Etat », c'est précisément des «embarras de la sociologie » et non de l'Etat comme phénomène de la vie sociale. Dans la vie sociale, personne n'est embarrassé avec l'Etat, parce que toute personne qui y fait référence possède, au moment où elle s'y réfère, une idée

${ }^{33}$ Dominique Linhardt, «L'embarras de la sociologie avec l'Etat. Groupes sociaux et collectifs politiques au prisme de l'argument pluraliste », dans Laurence Kaufmann et Dany Trom, dir., Qu'est-ce qu'un collectif? Du commun au politique, Paris, Editions de l'EHESS, ("Raisons pratique », vol. 20)

${ }^{34}$ Sur la question du « missing-what » chez Garfinkel, voir Michael Lynch, Scientific practice and ordinary action: Ethnomethodology and social studies of science, Cambridge University Press, 1993, p. 270275.

${ }^{35}$ Voir Louis Quéré, , «Pour une sociologie qui 'sauve les phénomènes' », Revue du MAUSS, n²4 («Une théorie sociologique générale est-elle pensable ?»), 2004. 
à peu près précise de ce qu'elle y met (et si elle n'a pas d’idée précise ou si elle n'a aucune idée, elle ne fait tout simplement référence à rien, comme le soulignait Winch). En ce sens, l'Etat (comme beaucoup d'autres choses) possède une existence frégienne, c'est-à-dire une existence propositionnelle qui n'implique pas l'existence concomitante d'une entité pouvant être explorée en tant que telle ${ }^{36}$. Cette existence est, toutefois, constitutive d'un phénomène au même titre que s'il s'agissait d'une entité physique.

Nous retrouvons, en fait, la configuration triadique évoquée par Dominique Linhardt, où la relation entre deux individus est réalisée par la référence à une tierce entité. Des hautsfonctionnaires, qui se désignent comme des "serviteurs de l'Etat", ne servent pas effectivement l'Etat en tant qu'entité (comme ils serviraient le roi, s'il y en avait un), mais cette référence soutient un jeu de catégorisation impliquant des droits et des devoirs par lesquels se réalise effectivement ce que la communauté à laquelle ils appartiennent considère comme étant le «service de l'Etat». Un diplomate, qui reçoit les condoléances d'un ministre dans un pays où un militaire français est mort en opérations, n'est pas l'Etat français (pas plus que le ministre n'est son propre Etat), mais la référence à cette entité sert de guide aux relations que l'un et l'autre doivent avoir dans ces circonstances, elle établit un ensemble de droits et de devoirs localement pertinents. Si, d'aventure, de telles condoléances n'étaient pas formulées, une série d'inférences parfaitement réelles et conséquentielles pourraient en procéder, qui tiendraient à l'attitude de l'«Etat» en question à l'égard de la «France ». Au total, si l'on considère la suite des interactions, c'est tout comme si l'Etat existait, puisque les interactants s'orientent par rapport à lui et se conduisent, donc, comme s'il était bien réel en tant qu'entité. En d'autres termes, son existence n'a pas besoin d'être instanciée pour avoir des effets. Dans le monde de la vie - qui n'est peut-être pas toujours celui des sciences sociales et politiques -, sa réalité est constituée de ce seul mécanisme. L'embarras ne commence, en fait, que lorsqu'on veut accéder à l'Etat indépendamment des actions qui s'y réfèrent ou que l'on prétend lui attribuer des caractéristiques stables, bref, lorsque le «scientifique » se met en tête de l'instancier pour l'analyser plus commodément. Cet embarras n'est pas un obstacle substantiel pour accéder aux phénomènes, dans la mesure où ceux-ci sont directement accessibles, mais il tend à rendre opaque ce qui pourtant s'offre à notre appréhension ordinaire de l'ordre usuel des choses.

Le fond du problème est que l'attitude théorisante est, si l'on peut ainsi dire, chevillée au corps de la démarche disciplinaire et que l'on croit toujours plus ou moins que parler des théories revient à parler des phénomènes. Cette démarche tient pour acquis que les phénomènes dont elle traite existent dans les termes dont elle en parle. S’ils existaient dans ces termes, comme des entités existentiellement indépendantes des humains, ou s'il suffisait d'ajuster ces termes pour en parler convenablement, comme nous le fait accroire la conception la plus répandue de l'épistémologie, la théorie serait effectivement le bon moyen pour discuter des choses elles-mêmes. Le problème est, toutefois, comme l'a souligné Winch avec force, que tout un pan, et probablement le plus important, des activités humaines ne nous est accessible qu'à partir et dans la perspective humaine qui est inexorablement la nôtre. C'est, en effet, l'activité conceptuelle des humains qui produit les phénomènes de manière telle qu'ils sont dotés d'un sens. Le problème de l'approche théorisante est qu'elle procède à une mise en abîme (une compréhension d'une compréhension) qui obscurcit les phénomènes mêmes qu'elle prétend clarifier. Elle remplace la conceptualisation des gens du monde ordinaire par la conceptualisation des observateurs. Il en découle que le domaine de la politique devient problématique, que l'on discute du moment où commence la politisation ou des embarras que pose l'Etat. Ce sont là des sujets d'incertitude pour la théorie, alors que ça n'en sont pas dans le

${ }^{36}$ Le fond de la question est que l'on recherche toujours, derrière la référence, un existant stable et descriptible de manière indépendante. Mais, quand je parle du «Capitaine Fracasse », il n'y a rien de stable derrière cette dénomination. On peut parler du «Capitaine Fracasse » en ayant en tête le roman de Théophile Gautier ou le film de Pierre Gaspard-Huit ou l'acteur qui interprète le personnage dans le film, c'est-à-dire Jean Marais. De quoi je parle quand je parle du «Capitaine Fracasse » est dépendant de ce que j'ai en tête quand j'en parle et de ce qui est disponible dans mon environnement pour documenter ce qu'est le «Capitaine Fracasse » dont je parle. Ce n'est pas dépendant de l'existence d'un «Capitaine Fracasse ». Je ne peux donc tout simplement pas décrire le «Capitaine Fracasse » en dehors de ce que je mets (intuitivement, implicitement) dans la référence que je fais, sauf à inventer à mon tour un personnage à partir d'un travail documentaire. En d'autres termes, les existences propositionnelles se suffisent à elles-mêmes. Tenter de les approfondir, c'est en inventer d'autres. 
monde ordinaire : tout un chacun sait différencier ce qui est politique de ce qui ne l'est pas, comme il sait quand il fait de la politique et quand il n'en fait pas ; enfin, personne n'est embarrassé lorsqu'il s'agit de désigner l'Etat. Ainsi, l'incertitude n'apparait-elle que quand on prétend voir "par-dessus » les gens, en surplomb du monde et de son épaisseur phénoménologique, alors qu'il suffirait, simplement et grossièrement, comme le souligne Wittgenstein, de regarder et voir ce qui git sous nos yeux.

Affirmer, après Phil Hutchinson, Rupert Read et Wes Sharrock commentant l'actualité de Peter Winch, qu'«il n'existe pas quelque chose comme une science sociale » ne revient pas à dire qu'il n'existe pas de phénomènes sociaux ou de phénomènes politiques qui puissent être étudiés en tant que tels. Pour en rester au politique, celui-ci existe comme objet de préoccupation pour les membres de la société et, partant, comme objet d'étude pour les politistes. Ce qui pose problème, c'est prétendre le repérer et l'analyser par-dessus l'épaule des gens. Contrairement aux entités non humaines des sciences de la nature, les êtres humains, eux, nomment et qualifient le réel. Il n'est donc pas possible de dire ce qu'ils font indépendamment de la prise en compte de leur point de vue, c'est-à-dire des «méthodes » par lesquelles le monde et leurs actions leurs sont mutuellement intelligibles. Inversement, les phénomènes naturels ne viennent à l'intelligibilité que par l'intermédiaire des conceptualisations des observateurs. Sans la conceptualisation, il s'agit de faits bruts. La chaise que je vois n'est composée d'électrons que parce que les physiciens me l'ont dit. Par elle-même, la chaise ne me dit rien. Pareillement, la girafe ne me dit pas qu'elle est un mammifère. Ce sont les savants qui le font. Dans le monde social, au contraire, les phénomènes sont déjà conceptualisés. Ce qui est une conceptualisation du premier degré, dans le monde naturel, y devient donc une conceptualisation du second degré, non pas du fait que les "scientifiques" changent de posture, mais du fait que les «êtres » auxquels ils appliquent leurs analyses sont ou ne sont pas conceptualisants (ou dotés d'intentionnalité manifeste). La collusion de la posture disciplinaire et du scientisme pousse donc les observateurs à substituer une conceptualisation qui leur est propre à la conceptualisation inhérente à l'activité routinière des gens ordinaires et les entraîne à s'enfermer dans des discussions internes portant sur cette "sophistication de second degré » plutôt qu'à regarder les phénomènes. Ils en finissent, souvent, à ne plus voir ceux-ci qu'à travers le prisme de leurs théories, la réalité n'en étant plus conçue que comme un cas d'application. A l'encontre de cette tendance assez largement dominante, quoiqu'il en soit de la diversité de ses expressions, Winch nous propose de réviser nos pratiques d'analystes des phénomènes sociaux en abandonnant l'idée de faire de la «science». Si les disciplines demeurent, dans ce cas, c'est en tant qu'elles circonscrivent un domaine d'intérêt et non qu'elles prétendent circonscrire un domaine de réalité. L'aisance avec laquelle cette approche nous tire de pas mal d'embarras suggère, pour le moins, de la prendre en considération.

Jean-Noël Ferrié, CNRS-PACTE, Institut d'Etudes Politiques de Grenoble et CERI, Sciences-Po, Paris Baudouin Dupret, CNRS, Centre Jacques-Berque, Rabat 